This is a self-archived version of an original article. This version may differ from the original in pagination and typographic details.

Author(s): Long, Fangfang; Ye, Chaoxiong; Li, Ziyuan; Tian, Yu; Liu, Qiang

Title: Negative emotional state modulates visual working memory in the late consolidation phase

Year: 2020

Version: Accepted version (Final draft)

Copyright: (c) 2020 Taylor \& Francis

Rights: In Copyright

Rights url: http://rightsstatements.org/page//nC/1.0/?language=en

Please cite the original version:

Long, F., Ye, C., Li, Z., Tian, Y., \& Liu, Q. (2020). Negative emotional state modulates visual working memory in the late consolidation phase. Cognition and Emotion, 34(8), 1646-1663. https://doi.org/10.1080/02699931.2020.1795626 


\title{
Negative emotional state modulates visual working memory in the late consolidation phase
}

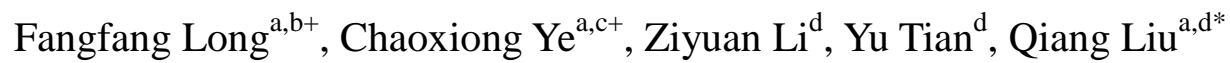

a. Institute of Brain and Psychological Sciences, Sichuan Normal University, Chengdu, China, 610000

b. Department of Psychology, Nanjing University, Nanjing University, China, 210023

c. Department of Psychology, University of Jyvaskyla, Jyväskylä, Finland, 40014

d. Research Center of Brain and Cognitive Neuroscience, Liaoning Normal University, Dalian, China, 116029

+ Fangfang Long and Chaoxiong Ye contributed equally to this work and should be considered as co-first authors.

This is an accepted manuscript of an article published [online] by Taylor \& Francis in Cognition and Emotion on $19^{\text {th }}$ July 2020, available online: https://www.tandfonline.com/doi/full/10.1080/02699931.2020.1795626

\section{* Correspondence to:}

Qiang Liu, PhD.

Professor, Institute of Brain and Psychological Sciences,

Sichuan Normal University, Chengdu, 610000, China

Telephone: +8613332220573

E-mail: lq780614@163.com 


\begin{abstract}
Although a considerable literature has grown up around the interactions between emotional state and visual working memory (VWM) performance, the mechanism underlying the impact of the negative emotional state on VWM remains unclear. The present study aimed to test whether the influence of emotional state is related to the early phase or late phase of VWM consolidation process. Across three experiments, we found that the negative emotional state did not affect VWM performance when the presentation time of stimuli was short. However, when the presentation time was long, the negative emotional state increased the VWM precision and reduced the VWM number. According to the two-phase model proposed by Ye et al. (2017), the results suggested that negative emotional state could affect the late phase of resource allocation in VWM consolidation process, but it has no impact on the early consolidation phase. The findings from this study make important contributions to the current literature regarding the emotional modulation of VWM.
\end{abstract}

Keywords: negative emotional state, visual working memory, resource allocation, two-phase model, late consolidation phase 


\section{Introduction}

Visual working memory (VWM) is a limited capacity system that can temporarily store visual information for other advanced cognitive processes (Luck \& Vogel, 2013; Ma, Husain, \& Bays, 2014), which provides individuals with a dynamic processing storage platform (Fukuda, Vogel, Mayr, \& Awh, 2010; Ikkai, McCollough, \& Vogel, 2010). Recent studies have suggested that the VWM process was influenced by the emotional state induced by negative stimuli (Figueira et al., 2017; Spachtholz, Kuhbandner, \& Pekrun, 2014; Xie \& Zhang, 2016). However, the impact of the negative emotional state on VWM remains unclear.

Several previous studies supported the idea that the negative emotional state would impair working memory. For example, Spies, Hesse, and Hummitzsch (1996) used classical measurements of memory span, speech rate, and reading span to show that the negative emotional state reduced working memory capacity. Figueira et al. (2017) used a change detection task to investigate the impact of the negative emotional state on VWM. By using the ERP technique and adopting a component named contralateral delay activity (CDA), which is widely held to be an indicator of VWM storage (Luria, Balaban, Awh, \& Vogel, 2016; Vogel \& Machizawa, 2004), Figueira et al. (2017) found that participants could store four colors in VWM under the neutral emotional state, whereas only two colors could be kept under the negative emotional state. These results have also been repeated by a follow-up study (Figueira et al., 2018). These ERP results have suggested that the negative emotional state reduces VWM capacity.

However, not all evidence supports that the negative emotional state could only reduce VWM performance. For example, by using movie clips to induce the participants' emotions, researchers found that in a change detection task both negative and positive emotion states could enhance VWM performance in the high VWM capacity group but reduce VWM performance in the low VWM capacity group (Y. Zhang, Zhang, \& Liu, 2017). Thus, it may be difficult to observe the effect of emotional state on VWM performance across all participants in the change detection task. In addition, it is worth noting that there was no significant difference in the VWM behavioral performance between the neutral emotional state and negative emotional state conditions in Figueira et al. (2017)'s study. The contradiction between the ERP and behavioral results in Figueira et al. (2017) may also be attributed to the insensitivity of the behavioral index (accuracy) to the emotional state in the change detection task. The accuracy result is influenced by both the number and precision of the representations stored in VWM. Previous studies found that there was a trade-off ratio between the VWM number and precision. The number of stored items in VWM decreases with the increase of VWM precision, and vice versa (Barton, Ester, \& Awh, 2009; Bays, Catalao, \& Husain, 2009; Machizawa, Goh, \& Driver, 2012). In this case, the adjustment in the trade-off ratio does not necessarily lead to a change of accuracy. Although the accuracy decreases with the decrease of the VWM number, it also increases with the increase of the VWM precision. Thus, if the VWM number reduces 
while the VWM precision increases, the change of the accuracy results may not be observed in the change detection task. It may explain why Figueira et al. (2017) could not find the effect of the negative emotional state on the VWM accuracy. Since the CDA component mainly reflects the number of stored representations rather than VWM precision (Gao, Yin, Xu, Shui, \& Shen, 2011; but see Sessa, Luria, Gotler, Jolicoeur, \& Dell'Acqua, 2011), the contradiction between the ERP and behavioral results implied that while the negative emotional state reduced VWM number, it might also improve the precision of representations in VWM. Therefore, the negative emotional state may not impair VWM, but alter the trade-off ratio between precision and number of VWM.

This possibility was supported by a previous study. By using a recall task, researchers can use a model-fitting method to separate the VWM number index and VWM precision index (W. Zhang \& Luck, 2008). For instance, Spachtholz et al. (2014) investigated the impact of the negative emotional state on visual sensory memory (VSM) and VWM by using recall tasks. They found that the negative emotional state reduced the number of items stored in VSM and VWM compared with the neutral emotional state, but surprisingly, the precision of VSM and VWM increased with the decline in memory number. That is, the negative emotional state altered the trade-off ratio between precision and number in VSM and VWM. Therefore, this study provided evidence for the functional influence of the negative emotional state on VWM, which was to improve VWM precision by sacrificing the VWM number effectively.

However, even with the recall task, not all studies supported that the negative emotional state affected the trade-off ratio between precision and number in the VWM. Xie and Zhang (2016) found that the negative emotional state could improve VWM precision even without sacrificing the VWM number. That is, in their study, they only found a positive effect of the negative emotional state on VWM. Thus, although the impact of the negative emotional state on VWM remains unclear, recent studies suggest that the negative emotional state could at least increase the precision of items stored in VWM.

The processes of memory stimuli in VWM task include two important parts: sensory encoding process and consolidation process. The sensory encoding process refers to the transformation of visual information into sensory representations, while the consolidation process refers to the transformation of these sensory representations into stable VWM representations. Some researchers suggested that VWM consolidation is actually a process in which individuals allocate VWM resources ${ }^{1}$ to their

\footnotetext{
1 A discrete slot-based theory (Balaban, Fukuda, \& Luria, 2019; Luck \& Vogel, 1997; Vogel, Woodman, \& Luck, 2001; W. Zhang \& Luck, 2008) and a continuous resource theory (Bays et al., 2009; Bays \& Husain, 2008; Bays, Wu, \& Husain, 2011; Wilken \& Ma, 2004) have been proposed for the nature of mental commodity that supports VWM storage. The term resources used in our paper is actually a neutral term. This term has the same meaning as the term mental commodity in Suchow, Fougnie, Brady, and Alvarez (2014)'s study. It can either refer to the "slots" based on the framework of the discrete slot-based theory, or refer to the "continuous resources" based on the framework of the continuous resource theory. In our paper, the term resources is only used to improve the coherence, but it does not mean that we prefer one of these two theories.
} 
consolidated representations (Becker, Miller, \& Liu, 2013; Hao, Becker, Ye, Liu, \& Liu, 2018; Liu \& Becker, 2013; Mance, Becker, \& Liu, 2012; Miller, Becker, \& Liu, 2014). A recent proposed two-phase VWM resource allocation model has explained the resource allocation in the VWM consolidation process (Ye et al., 2017; Ye et al., 2019). The model has suggested that resource allocation is a two-phase process when individuals consolidate visual information into VWM. Since individuals are not able to anticipate the length of presentation time of external visual information in the early phase of VWM consolidation, it is conducive to their survival to maintain more visual items even with low precision. Thus, individuals would only automatically allocate VWM resources to as many representations as possible in the early phase. This leads to a fixed trade-off ratio between VWM precision and number in the early phase. That is, the trade-off ratio in the early phase is determined by the stimulus-driven factor (set size of memory stimuli). When individuals consolidate as many visual items as possible, the early phase of VWM resource allocation is completed. At this time, if the external visual information still exists, the individuals could effectively consolidate more new information into VWM for creating representations with higher precision according to their bias or strategy. For example, if the task requires participants to memorize the stimulus with high VWM precision (e.g., recall task), in the late phase of VWM consolidation participants could reduce the VWM number to free up VWM resources, and then use the resources to improve the memory precision of the remaining representations. That is, in the late phase of VWM consolidation, individuals can voluntarily reallocate VWM resources to trade off the VWM number and precision. At this phase, the trade-off ratio can be adjusted by factors other than the stimulus-driven factor. Thus, the length of presentation time of visual stimuli affects whether individuals can enter the late phase and whether they can adjust the trade-off ratio of precision and number according to their preference.

It is natural to ask about the mechanism underlying the improvement of VWM precision under the negative emotional state. There are two possibilities to explain this phenomenon. The first possibility is that the negative emotional state mainly affects resource allocation in the early phase of VWM consolidation. In the early VWM consolidation, individuals would create as many representations as possible with low precision. In the negative emotional state, individuals may consolidate the representation more effectively. For example, a negative emotional state may improve the utilization efficiency of VWM resources. The minimum memory precision of the representations formed in the early consolidation phase under a negative emotional state was higher than the one under the neutral emotional state. That is, in the negative emotional state, individuals form VWM representations with higher precision in the early consolidation phase, which leads to the enhancement of their VWM performance. The second possibility is that the negative emotional state mainly affects the voluntary VWM resource allocation in the late phase of VWM consolidation, instead of the minimum memory precision of the representations in the early consolidation phase. The precision of representation formed in the early consolidation phase under different emotional states is the same. That is, the early phase of VWM 
consolidation in different emotional states have the same effect on VWM performance. Thus, the influence of the negative emotional state on VWM performance mainly related to the later process of VWM consolidation. After individuals enter the late consolidation phase, they would reallocate VWM resources and tend to allocate more VWM resources to few representations to improve VWM precision in the negative emotional state.

Therefore, in the present study, based on the framework of the two-phase model (Ye et al., 2017; Ye et al., 2019), we tested whether the effect of negative emotional state on VWM performance related to the early phase or late phase of VWM consolidation process. If the influence of the negative emotional state on VWM performance related to the early consolidation phase and created high-precision VWM representations, it was expected that the negative emotional state could improve the VWM precision even when the presentation time of stimuli was not enough for the individuals to enter the late phase of resource allocation. On the contrary, if the influence of a negative emotional state on VWM mainly occurred in the late consolidation phase, it was expected that negative emotional state had no impact on the trade-off ratio of VWM number and precision when the presentation time was short. However, the negative emotional state did alter the trade-off ratio between VWM number and precision when the presentation time of stimuli was long enough to allow the individual to enter the late phase of resource allocation in the consolidation process.

\section{Experiment 1}

The aim of Experiment 1 was to explore the impact of the negative emotional state on the number and precision when the presentation time of stimuli was insufficient to allow participants to enter the late phase. Participants were asked to remember four orientations during $200 \mathrm{~ms}$ presentation time, which was too short to adjust the trade-off ratio between the VWM number and precision according to their preference (Ye et al., 2017). Thus, if the result showed that the VWM precision in the negative emotional state was higher than that in the neutral emotional state, it suggested that the negative emotional state improved the VWM precision in the early phase of the consolidation process. On the contrary, if we could not observe significant differences in VWM number and precision between the neutral and negative emotional state conditions, we would further examine the effect of negative emotional state on VWM performance in Experiment 2.

\section{Method}

Participants. Thirty-three undergraduate students from Liaoning Normal University (20 women, 33 right-handed, 19-23 years old) volunteered to participate in this experiment for compensation at a rate of $\$ 3 /$ hour. They reported having a normal color vision and normal or corrected-to-normal visual acuity, and no history of neurological problems. Each participant provided written informed consent before the 
experiment. All procedures were conducted following the Declaration of Helsinki (2008) and were approved by the ethics committee of Liaoning Normal University (the approval number: 1NNUIRB1710). Three participants were excluded due to a large guess rate $(>60 \%)$, such that the results reported below were based on data from 30 participants.

We based our sample size on a previous study that compared VWM performance of neutral and negative emotion by Spachtholz et al. (2014). Based on the method proposed by Thalheimer and Cook (2002) for estimating effect sizes from t-tests, we estimated that their effect size was 0.608 for that comparison. To determine our sample size we assumed that our effect size is the same with their effect size, and calculated the sample size required to yield a power of .85 given $\alpha=.05$ for a paired-sample t-test (Faul, Erdfelder, Lang, \& Buchner, 2007). This calculation showed that the minimum required sample size was 18 .

Stimuli. The experiment was programmed using E-Prime 2.0. Sixty emotional images were selected from the International Affective Picture System (IAPS) for each of the two emotional state conditions (neutral and negative). Each selected image had a high agreement rate in emotion categorization. The normative valence ratings (mean [SD]) were $2.45(0.73)$ for negative condition, and 5.51(0.66) for neutral condition. The normative arousal ratings were $5.75(0.80)$ for the negative condition and $3.32(0.80)$ for the neutral condition. T-test analyses revealed significant differences in valence, $\mathrm{t}(118)=24.02, \mathrm{p}<.001$, and arousal, $\mathrm{t}(118)=16.62, \mathrm{p}<.001$, across these two image sets.

The memory stimuli were the same as those used in Ye et al. (2017)'s study, which were sinusoidal gratings (contrast, 0.7; spatial frequency, 3 cycles/degree) in a circular aperture (size, $0.9^{\circ}$ ) presented on a gray background. The orientation of each stimulus was randomly selected from 90 possible angles evenly spaced from $0^{\circ}$ to $180^{\circ}$, with the orientations separated by at least $12^{\circ}$. The gratings could be presented in four possible locations located at the corners of an imaginary square (eccentricity, $3^{\circ}$ ). The experimental environment was similar to that in Ye et al. (2017)'s study, the stimuli were presented on a 19 inch CRT monitor $(1280 \times 768$ pixel), and participants viewed the display at a distance of $60 \mathrm{~cm}$ in a dark room.

Procedure. In each trial, participants needed to complete two different tasks. As depicted in Figure 1, each trial started with a valence judgment task for emotion induction, followed by an orientation recall task for VWM. The valence judgment task in our study was similar to the one in Xie and Zhang (2016)'s study. An IAPS image was presented at the center of the screen for 4,000 ms, and participants reported its valence on the 9-point Self-Assessment Manikin (SAM) scale using a computer mouse. IAPS images were originally colored but were converted to gray-scale to minimize potential interference with the subsequent VWM task. The valence judgment task was followed by the orientation recall task. After an 800-ms fixation, 
four oriented gratings were presented for $200 \mathrm{~ms}$, followed by a $1000 \mathrm{~ms}$ retention period. Then a location cue (a $1^{\circ}$ square outline) appeared in one of the stimulus locations, along with an adjustable probe grating (presented at fixation). By using a computer mouse, participants adjusted the orientation of the probe to match that of the cued grating and then finalized adjustments until they were satisfied. The emotional state (negative and neutral) conditions were blocked with their order counterbalanced across participants. Each image within each condition was randomly selected and presented twice for the valence judgment task, yielding 120 trials for each condition.

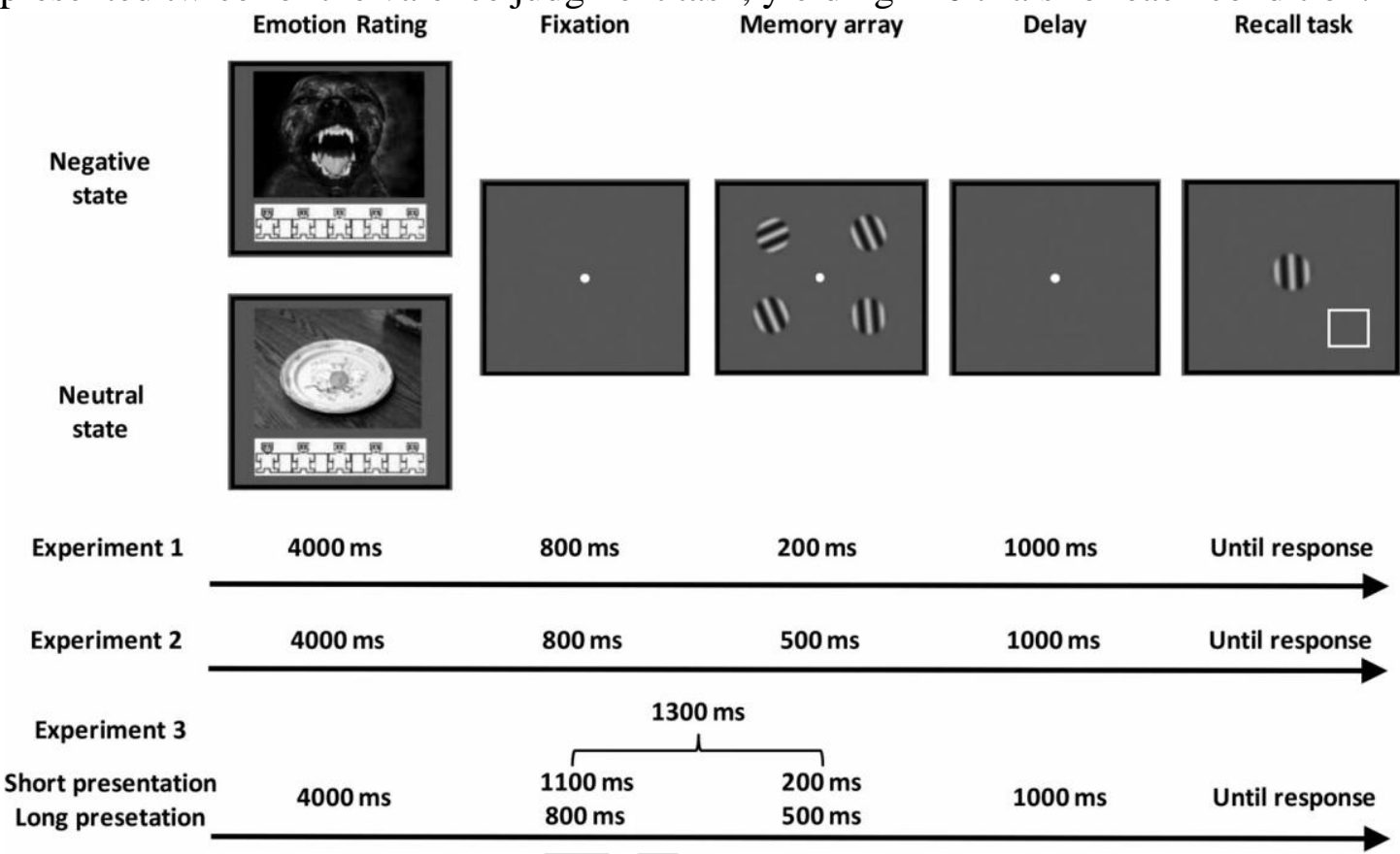

Figure 1. Trial structure of Experiment 1, 2 and 3. A negative emotional state condition and a neutral emotional state condition with different presentation time periods of memory array (200 ms for Experiment 1; $500 \mathrm{~ms}$ for Experiment 2; $200 \mathrm{~ms}$ or $500 \mathrm{~ms}$ for Experiment 3) were illustrated.

Data analysis. For each trial, the offset (error) in the recalled orientation was calculated by subtracting the reported orientation setting from the target orientation. We adopted the standard mixture model (W. Zhang \& Luck, 2008) to fit the data using the MemToolbox (Suchow, Brady, Fougnie, \& Alvarez, 2013). The standard mixture model assumed that performance on the orientation recall task was determined by a mixture of two types of trials. On a proportion of trials, participants did not consolidate the items into VWM but guessed, with the reported orientation conforming to a uniform random distribution. On the remaining trials, participants successfully consolidated the items into VWM, which contained a noisy representation of the target orientation, modeled by a Von Mises distribution. The model allowed us to estimate the number of items stored in VWM $(\mathrm{K})$ as well as the precision of the VWM representation (SD). The $\mathrm{K}$ value was calculated by multiplying the set size with the probability of successful retrieval (i.e., 1- guess rate). SD was the circular standard deviation of a Von Mises distribution, which was inversely related to the VWM precision. We fitted the model to individual participant 
data in each condition and used a paired t-test to compare the model parameters at the group level to assess statistical significance between conditions (negative and neutral). In order to ensure that the chosen model can better fit the data, we compared the goodness-of-fit of the standard mixture model and the swap model by using the MemToolbox to compute the AIC (Suchow et al., 2013). The swap model assumes that besides the two types of trials in the standard mixture model, there was a third trial type in which participants reported the non-target orientation (Bays et al., 2009). The lower AICs were found for the standard mixture model, which suggested that the standard mixture model has better goodness-of-fit than the swap model. We found that the standard mixture model was most likely for 23 of 30 participants in Experiment 1, 24 of 30 participants in Experiment 2, and 23 of 30 participants in Experiment 3.

We also calculated the proportion of the experimental effect on $\mathrm{K}$ and SD values, assessed as $\left[\left(\mathrm{K}_{\text {Negative }}-\mathrm{K}_{\text {Neutral }}\right) / \mathrm{K}_{\text {Average }}\right]$ for effect on $\mathrm{K}$ and $\left[\left(\mathrm{SD}_{\text {Negative }}-\right.\right.$ $\left.\mathrm{SD}_{\text {Neutral }} / \mathrm{SD}_{\text {Average }}\right]$ for effect on $\mathrm{SD}$. A positive value of the experimental effect on $\mathrm{K}$ indicates that the proportion of negative emotional state effect can increases VWM number, while a negative value of it represents that the proportion of negative emotional state can reduce VWM number. On the contrary, a positive value of the experimental effect on SD indicates that the proportion of negative emotional state effect can decline VWM precision, while a negative value represents that the proportion of negative emotional state effect can increase VWM precision. Similar methods for calculating the experimental effects had been used in Xie and Zhang (2017)'s study.

\section{Results and Discussion}

For the pleasantness rating, the scores of the two emotional states were compared with the Wilcoxon Signed Ranks Test. The results showed that the difference between the two groups was significant, $Z=-4.412, p<.001$, as depicted in Figure 3a.

We fitted the mixture model to the individual participant data in each condition (Figure 2). The results showed that for both the VWM precision index (SD) and VWM number index $(\mathrm{K})$, there was no significant difference in $\mathrm{SD}, t(29)=0.339, p$ $=.737$, Cohen's $d=0.077$, and $\mathrm{K}, t(29)=0.702, p=0.489$, Cohen's $d=0.116$, between two conditions (Figure 3b-c). 

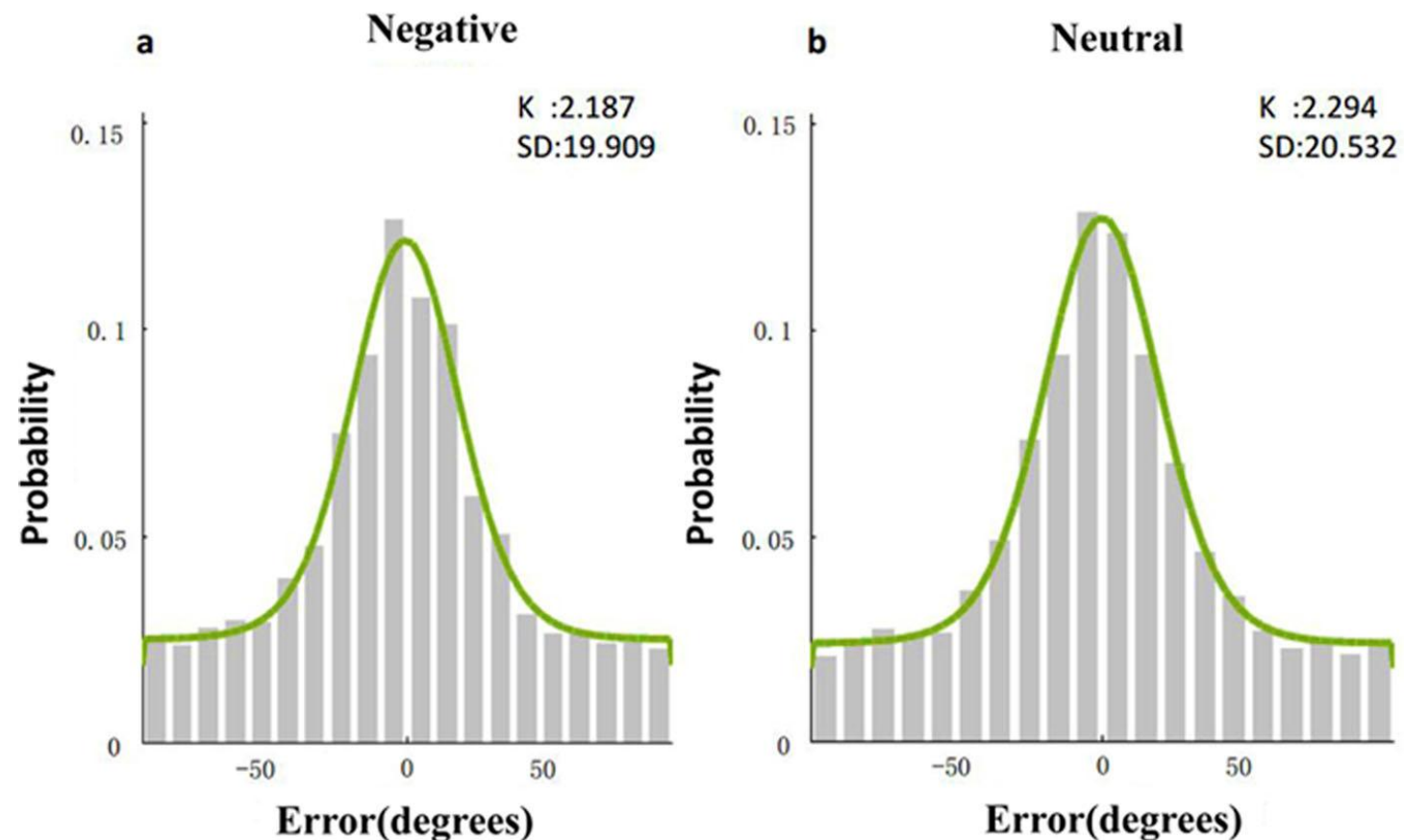

Figure 2. Results of fitting the mixture model to the aggregate data in Experiment 1. The graphs show probability density functions for the offset of responses in the (a) negative condition, and (b) neutral condition of Experiment 1. Model fits are illustrated by continuous lines. The memory number $(K)$ and circular standard deviation $(S D)$ are also shown for each condition.

For the experimental effects on $\mathrm{SD}\left[\left(\mathrm{SD}_{\text {Negative }}-\mathrm{SD}_{\text {Neutral }}\right) / \mathrm{SD}_{\text {Average }}\right]$ and $\mathrm{K}\left[\left(\mathrm{K}_{\text {Negative }}\right.\right.$ $-\mathrm{K}_{\text {Neutral }} / \mathrm{K}_{\text {Average }}$, the mean experimental effects were $-3.49 \%$ on $\mathrm{K}$ and $-4.40 \%$ on SD. The effects on SD and K had no significant difference from zero, $t(29)=0.434, \mathrm{p}$ $=.667$, Cohen's $d=0.112$ (effect on SD), $t(29)=0.516, p=.610$, Cohen's $d=0.133$ (effect on K). There was no significant difference in the experimental effects on SD and $\mathrm{K}, Z=-0.093, p=.926$. These results suggested that the negative emotional state had no effect on VWM precision and number.

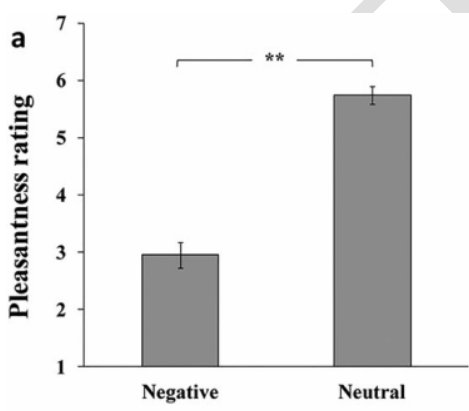

Emotional state

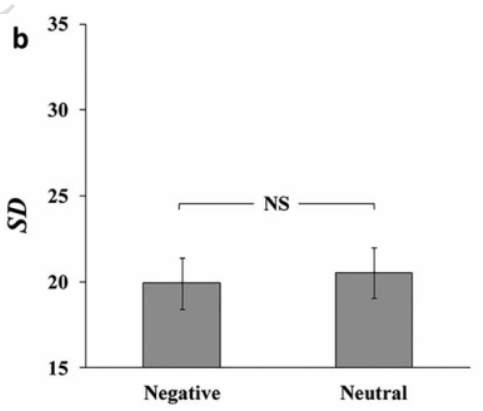

Emotional state

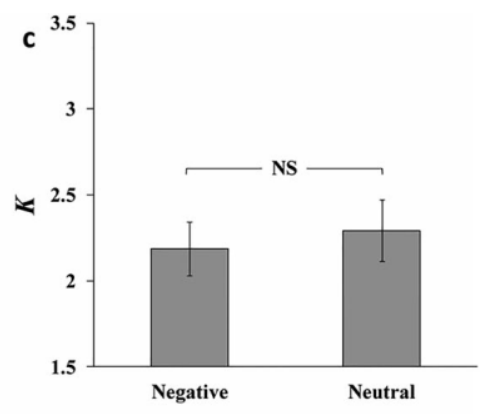

Emotional state

Figure 3. The results of Experiment 1. The (a) average pleasantness ratings of the International Affective Picture System (IAPS) images, (b) SD and (c) the mean number of items stored in memory $(K)$ are plotted separately for the negative and neutral emotional state conditions. No significant difference of $S D$ and $K$ was observed. Error bars are standard error of the mean. NS = non-significant; $* *=p<0.01$.

In Experiment 1, no difference was observed in VWM number and precision between 
different emotional state conditions. Thus, we failed to find the evidence to support that the influence of different emotional states on VWM performance was due to different early processes. There were two possibilities to explain the results. The first possibility was that, as we expected, the negative emotional state mainly actually affected the resource allocation in VWM processing. According to the study of Ye et al. (2017), the trade-off ratio between VWM number and precision only changed when the presentation time of stimuli was long enough. Previous ERP studies also showed that when the presentation time (100 to $200 \mathrm{~ms}$ ) was short, participants could not alter the trade-off ratio between VWM number and precision according to task requirements (He, Zhang, Li, \& Guo, 2015; Ye, Zhang, Liu, Li, \& Liu, 2014). Thus, the presentation time in Experiment 1 might be too short to allow participants to adjust the trade-off ratio between VWM number and precision. The second possibility was that the materials used in Experiment 1 failed to induce the negative emotional state to affect VWM process. Since the null result was insufficient to reject the hypotheses, we would further test them in Experiment 2.

\section{Experiment 2}

As explained above, the two-phase model suggested that the trade-off ratio between number and precision could be adjusted when presentation time was long enough (Ye et al., 2017; Ye et al., 2019). Thus, in Experiment 2, we used the same procedure as Experiment 1 but extended the presentation time of memory stimuli from $200 \mathrm{~ms}$ to $500 \mathrm{~ms}$, and tested whether the negative emotional state had an impact on the number and precision of VWM representations according to their preference when the presentation time was long enough.

\section{Method}

Participants. A new sample of 32 undergraduate students from Liaoning Normal University (20women, 32 right-handed,18-23 years old) volunteered to participate in this experiment for compensation at a rate of $\$ 3 /$ hour. They reported having a normal color vision and normal or corrected-to-normal visual acuity, and no history of neurological problems. Each participant provided written informed consent before the experiment. All procedures were conducted following the Declaration of Helsinki (2008) and were approved by the ethics committee of Liaoning Normal University (the approval number: 1NNUIRB1710). Two participants were excluded due to a large guess rate $(>60 \%)$, such that the results reported below were based on data from 30 participants.

Stimuli, procedure, and data analysis. The design and procedure were identical to those of Experiment 1, except that we increased the presentation time from $200 \mathrm{~ms}$ to $500 \mathrm{~ms}$, as depicted in Figure 1.

\section{Results and Discussion}


For the pleasantness rating, the scores of the two emotional states were compared with the Wilcoxon Signed Ranks Test. The results showed that the difference between the two groups was significant, $Z=-4.782, p<.001$, as depicted in Figure 5a.

We fitted the mixture model to the individual participant data in each condition (Figure 4). The results showed that the VWM precision index (SD) in the negative emotional state condition was significantly lower than that in the neutral emotional state condition, $t(29)=2.508, p<.05$, Cohen's $d=0.46$, which suggested that the VWM precision in the negative condition was higher than that in the neutral condition. For the VWM number index $(\mathrm{K})$, there was a significant difference between the two emotional state conditions $t(29)=2.382, p<.05$, Cohen's $d=0.44$. VWM number in the negative condition was lower than that in the neutral condition (Figure 5b-c).
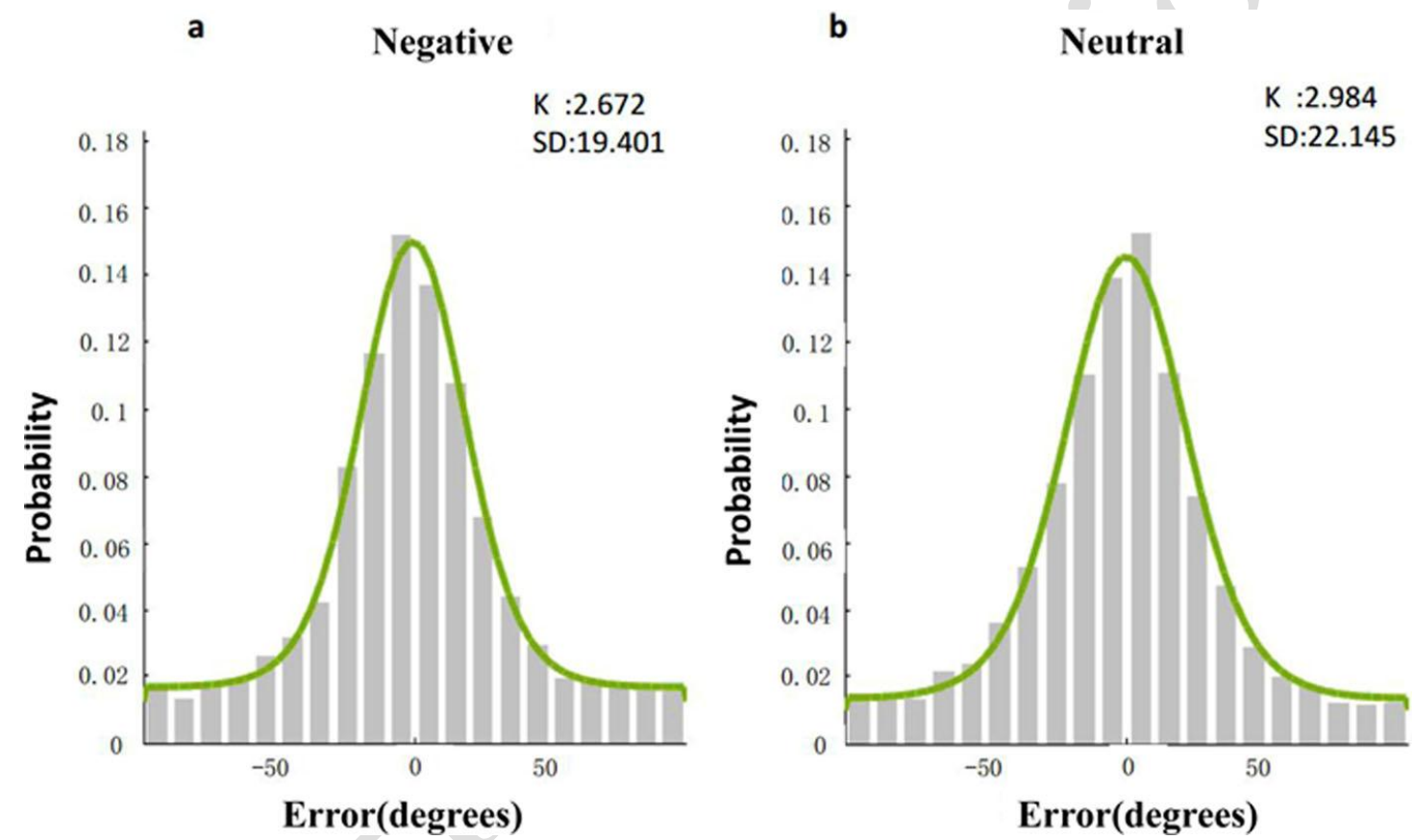

Figure 4. Results of fitting the mixture model to the aggregate data in Experiment 2. The graphs show probability density functions for the offset of responses in the (a) negative condition, and (b) neutral condition of Experiment 2. Model fits are illustrated by continuous lines. The memory number $(K)$ and circular standard deviation (SD) are also shown for each condition.

For the experimental effect on SD [ $\left.\left(\mathrm{SD}_{\text {Negative }}-\mathrm{SD}_{\text {Neutral }}\right) / \mathrm{SD}_{\text {Average }}\right]$ and $\mathrm{K}\left[\left(\mathrm{K}_{\text {Negative }}-\right.\right.$ $\left.\mathrm{K}_{\text {Neutral }}\right) / \mathrm{K}_{\text {Average }}$, the mean experimental effect was $-10.76 \%$ on SD and $-12.03 \%$ on $\mathrm{K}$, and both were significantly larger than zero, $t(29)=2.413, p<.05$, Cohen's $d=0.623$ $($ effect on K), $t(29)=2.573, p<.05$, Cohen's $d=0.664$ (effect on SD) There was no significant difference in the experimental effects on SD and $\mathrm{K}, Z=-0.093, p=.926$. Unlike the results of Experiment 1, these results suggested that the negative emotional state had significant effects on VWM precision (SD) and number (K). However, the proportion of the experimental effect on VWM precision was similar to that on VWM number. 

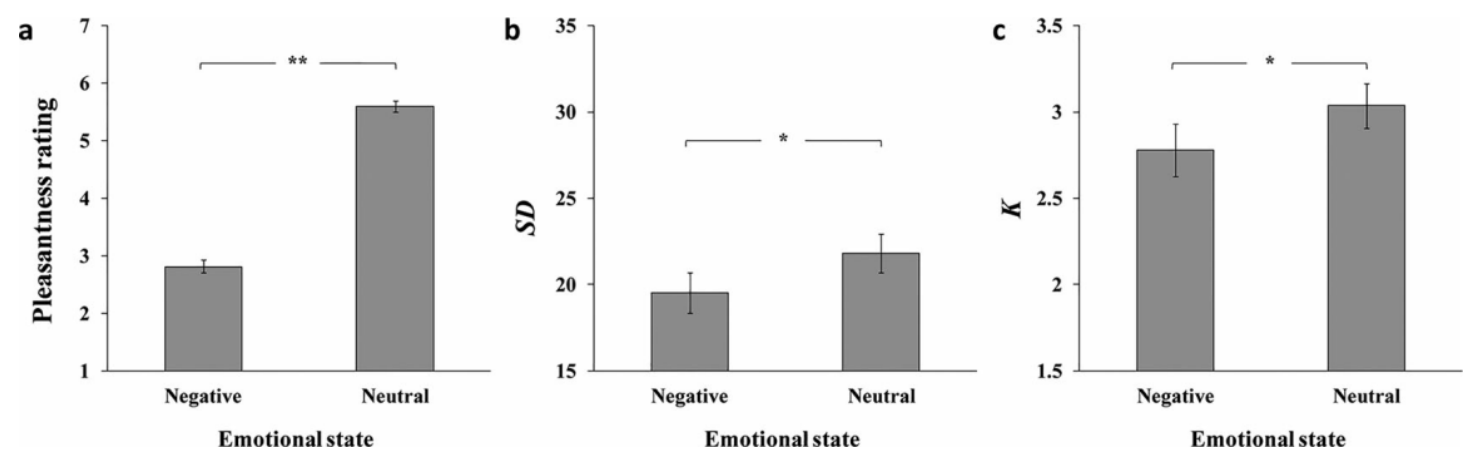

Figure 5. The results of Experiment 2. The (a) average pleasantness ratings of the International Affective Picture System (IAPS) images, (b) SD and (c) the mean number of items stored in memory $(K)$ are plotted separately for the negative and neutral emotional state conditions. Negative emotion improved VWM precision (i.e., smaller $S D$ ) and reduce VWM number (i.e., smaller K) Error bars are the standard errors of the means. $*=p<0.05 ; * *=p<0.01$.

In Experiment 2, the results showed that when the presentation time was long, participants remembered fewer items with higher precision in the negative emotional state condition compared with the neutral emotional state condition. These results rejected the possibility that the negative emotional state induced by materials in Experiment 1 could not have an impact on the VWM performance. These results suggested that the negative emotional state affected resource allocation in the late consolidation phase when the presentation time was long.

\section{Experiment 3}

In Experiment 1 and 2, we found that a negative emotional state did not affect VWM number and precision in a short presentation time of stimuli, but when the stimuli presented for a long time, the negative emotional state made participants memorize fewer items with higher precision in VWM compared to the neutral emotional state. However, due to the contamination by two potential factors, the current evidence might be still insufficient to support that the influence of the negative emotional state on VWM representations was related to resource reallocation in the late consolidation phase.

The first potential factor was that the emotion induction degree might be different in different experiments. We knew that it took time to induce a negative emotional state. In Experiment 1, after the disappearance of emotional pictures, an $800 \mathrm{~ms}$ fixation screen disappeared followed by the appearance of a 200-ms memory array. In this case, the interval between the offset of emotional pictures and the offset of memory array was $1000 \mathrm{~ms}$. However, in Experiment 2, after the disappearance of emotional pictures, an $800 \mathrm{~ms}$ fixation screen disappeared followed by the appearance of a 500-ms memory array. In this case, the interval between the offset of emotional pictures and the offset of memory array was $1300 \mathrm{~ms}$. The difference between the experimental designs made it possible for the participants to consolidate the memory stimuli after the emotional pictures disappeared 1000-1300 ms only in Experiment 2. 
If the participants needed about $1000 \mathrm{~ms}$ to induce their negative emotional state, it would lead us to observe the effect of negative emotional state on VWM performance only in Experiment 2, but not in Experiment 1. This possibility fitted well with our current results.

The second potential factor was that there were individual differences in emotional experience between different experiments. In order to avoid the practice effect, we selected a new sample as participants in Experiment 2. This would lead to inevitable individual differences in emotional experience in different experiments. The difference in result pattern between Experiment 1 and 2 could be partly attributed to individual differences in emotional experience.

In Experiment 3, by using a within-subject design, we manipulated presentation time (200 ms and $500 \mathrm{~ms}$ ) and emotional state (neutral and negative). Moreover, in order to ensure that the participants had the same emotional inducing time when they memorized the memory array under different presentation time conditions, we made the interval between the offset of emotional pictures and the offset of memory array consistent under different presentation conditions. These controls could eliminate potential issues in Experiment 1 and 2. In Experiment 3, we expected that we were able to observe the same findings as Experiment 1 and 2.

\section{Method}

Participants. A new sample of 35 undergraduate students from Liaoning Normal University (22 women, 34 right-handed,18-25 years old) volunteered to participate in this experiment for compensation at a rate of $\$ 5 /$ hour. They reported having a normal color vision and normal or corrected-to-normal visual acuity, and no history of neurological problems. Each participant provided written informed consent before the experiment. All procedures were conducted following the Declaration of Helsinki (2008) and were approved by the ethics committee of Liaoning Normal University (the approval number: INNUIRB1710). Five participants were excluded due to a large guess rate $(>60 \%)$, such that the results reported below were based on data from 30 participants.

Stimuli, procedure, and data analysis. The design and procedure were similar to those of Experiment 1 and 2, except that the same group of participants needed to complete the experiments at the presentation time of $200 \mathrm{~ms}$ and $500 \mathrm{~ms}$. In addition, when the memory array was presented for $200 \mathrm{~ms}$, the interval between the picture and the memory item was $1100 \mathrm{~ms}$. When the memory item was presented for $500 \mathrm{~ms}$, the interval between the picture and the memory item was still $800 \mathrm{~ms}$ (Figure 1). In Experiment 3, the two emotional state conditions (negative and neutral) were blocked and the order of blocks was balanced across the participants. There were 120 trials for each presentation time (200 ms and $500 \mathrm{~ms}$ ), with a total of 240 trials per emotional state block which were fully randomized. The whole experiment included 480 trials in 
total.

\section{Results and Discussion}

For the pleasantness rating, the scores of the two emotional states were compared with the Wilcoxon Signed Ranks Test. The results showed that the difference between the two groups was significant, $Z=-4.782, p<.001$, as depicted in Figure 7a.

We fitted the mixture model to the individual participant data in each condition (Figure 6) and conducted an ANOVA by taking the emotional state condition (neutral emotional state vs. negative emotional state) and presentation time (200 ms vs. 500 $\mathrm{ms}$ ) on the SD and K parameter, respectively.

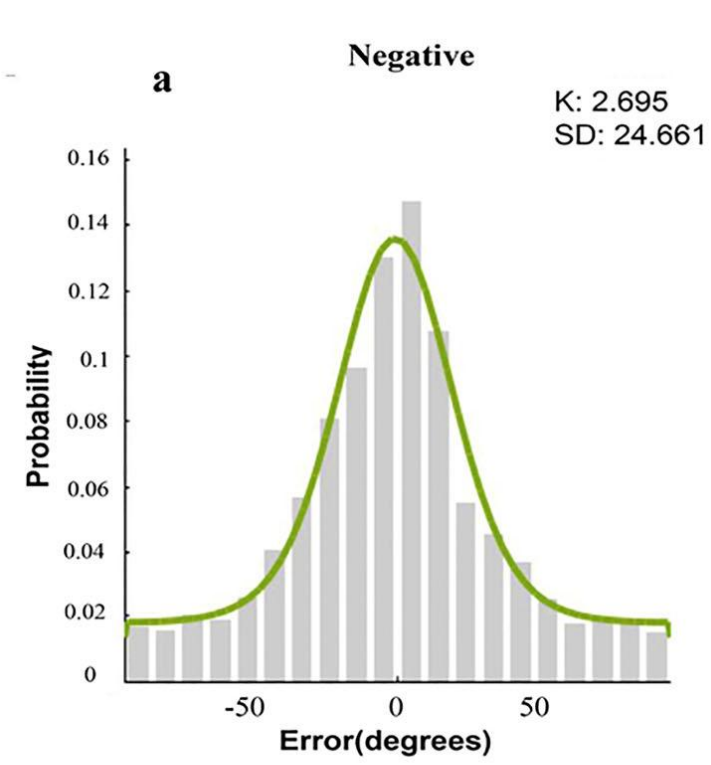

200ms
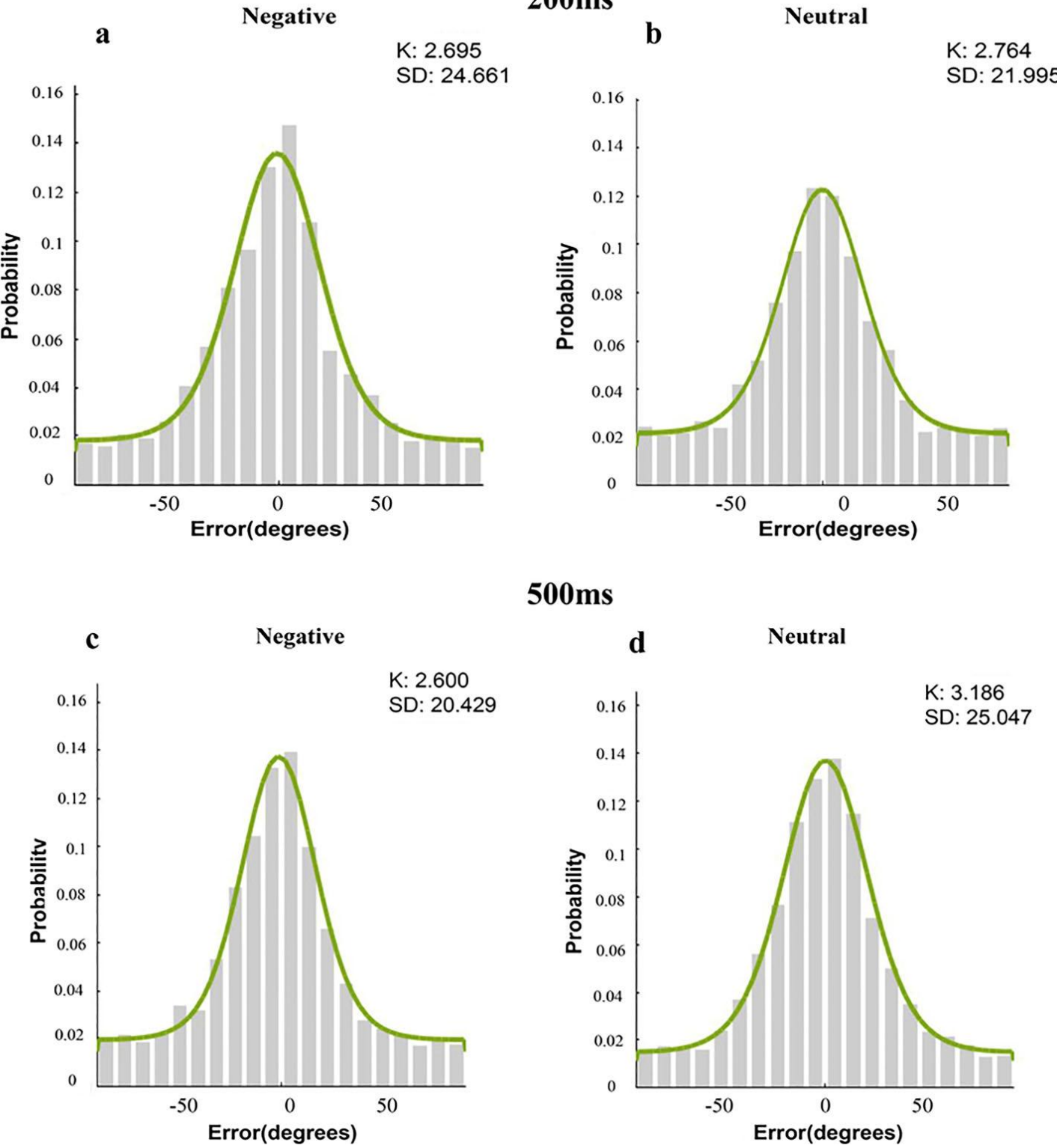

\section{$500 \mathrm{~ms}$}

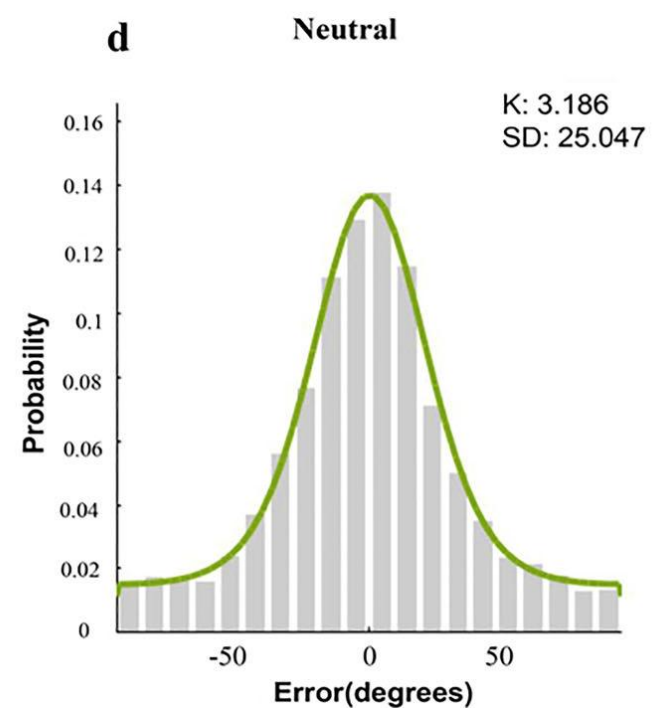

Figure 6. Results of fitting the mixture model to the aggregate data in Experiment 3. The graphs show probability density functions for the offset of responses in the (a) negative condition, and (b) 
neutral condition when the presentation time was 200 ms. The graphs show probability density functions for the offset of responses in the (c) negative condition, and (d) neutral condition when the presentation time was $500 \mathrm{~ms}$. Model fits are illustrated by continuous lines. The memory number $(K)$ and circular standard deviation $(S D)$ are also shown for each condition.

For SD, there was a significant interaction between emotional state and presentation time, $F(1,29)=5.194, p<.05, \eta^{2}=0.152$, but the main effects of emotional state, $F(1,29)=1.063, p=.311, \eta^{2}=0.035$, and presentation time, $F(1,29)=0.167, p=.686$, $\eta^{2}=0.006$, were not significant. The follow-up pairwise comparison showed that when the presentation time was $200 \mathrm{~ms}$, there was no significant difference in SD value between the neutral emotional state condition and negative emotional state condition, $t(29)=0.809, p=.425$, Cohen's $d=0.154$. However, when the presentation time was $500 \mathrm{~ms}$, the SD value was significantly higher in the neutral emotional state condition than in the negative emotional state condition, $t(29)=2.632, p<.05$, Cohen's $d=0.466$, (Figure 7b).

For K, there was a significant main effect of emotional state, $F(1,29)=6.269, p<.05$, $\eta^{2}=0.178$, and a marginally significant interaction between emotional state and presentation time, $F(1,29)=3.371, p=.077, \eta^{2}=0.104$, but the main effect of presentation time was not significant, $F(1,29)=1.311, p=.262, \eta^{2}=0.043$. The follow-up pairwise comparison showed that when the presentation time was $200 \mathrm{~ms}$, there was no significant difference in $\mathrm{K}$ value between the neutral emotional state condition and negative emotional state condition, $t(29)=0.479, p=.635$, Cohen's $d=$ 0.105. However, when the presentation time was $500 \mathrm{~ms}$, the $\mathrm{K}$ value was significantly higher in the neutral emotional state condition than in the negative emotional state condition, $t(29)=3.748, p<.01$, Cohen's $d=0.670$ (Figure $7 \mathrm{c}$ ). These results were the same as those in Experiment 1 and 2.
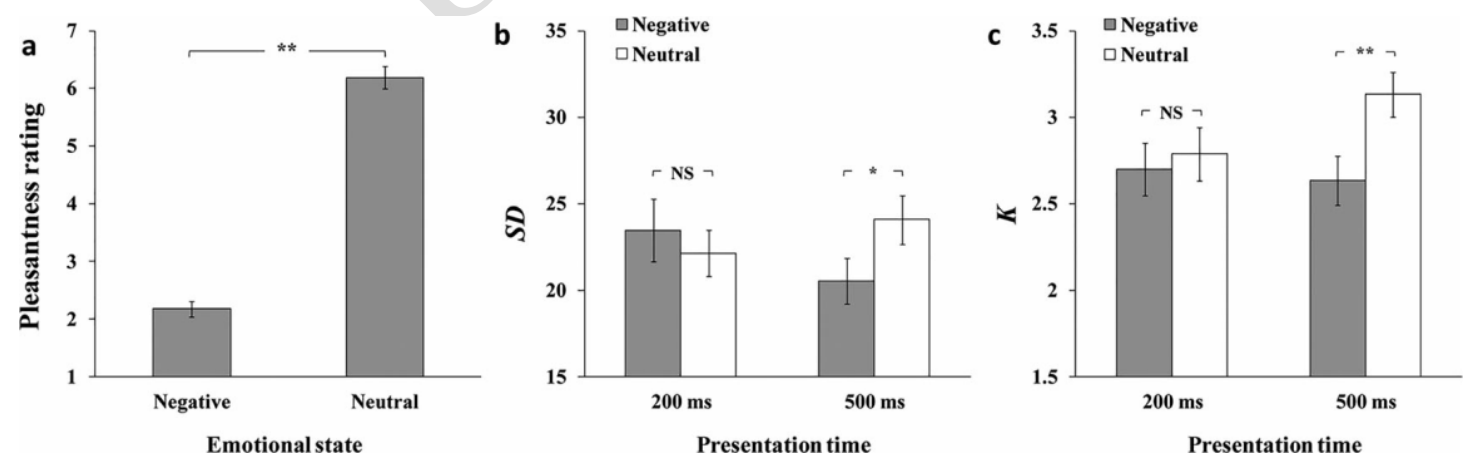

Figure 7. The results of Experiment 3. The (a) average pleasantness ratings of the International Affective Picture System (IAPS) images, (b) SD and (c) the mean number of items stored in memory $(K)$ are plotted separately for the negative and neutral emotional state conditions with short (200 ms) and long (500 ms) presentation time. No significant difference of SD and $K$ was observed for short presentation time condition. In contrast, negative emotion improved VWM precision (i.e., smaller $S D$ ) and reduce VWM number (i.e., smaller $K$ ) for long presentation time condition. Error bars are standard error of the mean. $*=p<0.05 ; * *=p<0.01$. 
When the presentation time was $200 \mathrm{~ms}$, the mean experimental effects were $1.60 \%$ on SD and $-3.76 \%$ on $\mathrm{K}$. The effects on SD and K showed no significant difference from zero, $t(29)=0.199, p=.844$, Cohen's $d=0.051$ (effect on SD), $t(29)=0.506, p$ $=.617$, Cohen's $d=0.130$ (effect on $\mathrm{K}$ ). There was no significant difference in the experimental effects on SD and $\mathrm{K}, \mathrm{Z}=-1.327, \mathrm{p}=.185$. When the presentation time was $500 \mathrm{~ms}$, the mean experimental effects were $-16.77 \%$ on SD and $-18.27 \%$ on $\mathrm{K}$, and both were significantly lower than zero, $t(29)=2.514, p<.05$, Cohen's $d=0.649$ (effect on SD), $t(29)=3.679, p<.01$, Cohen's $d=0.950$ (effect on K). There was no significant difference in the experimental effects on SD and $\mathrm{K}, Z=-1.183, p=.237$ (Figure 8). These results were the same as those in Experiment 1 and 2.

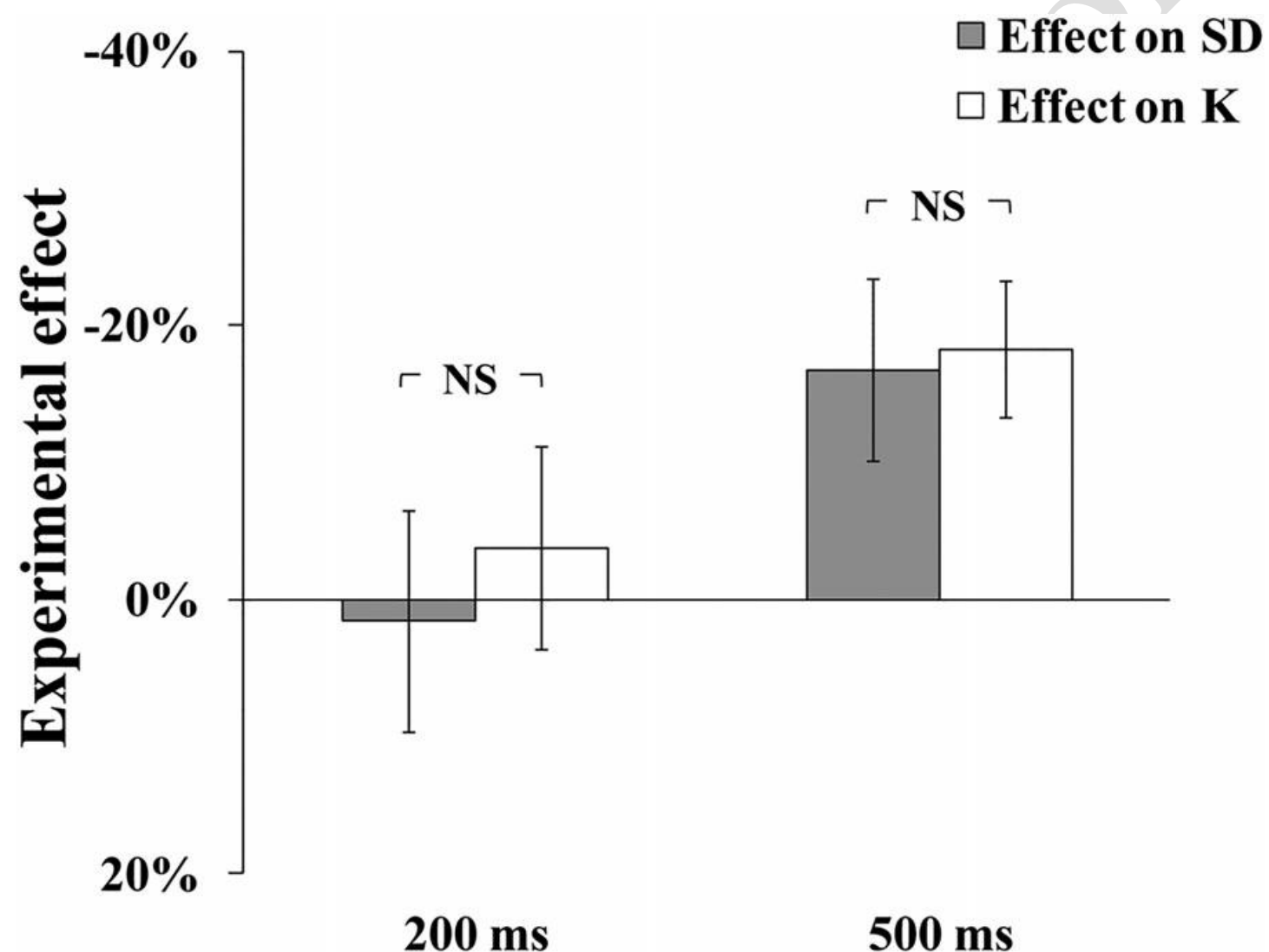

\section{Presatation time}

Figure 8. The results of the experimental effect on $S D\left[\left(S D_{\text {Negative }}-S D_{\text {Neutral }}\right) / S D_{\text {Average }}\right]$ and $K$ $\left[\left(K_{\text {Negative }}-K_{\text {Neutral }}\right) / K_{\text {Average }}\right]$ in Experiment 3. No significant difference of experimental effect was observed for both shrot and long presentation time.

In Experiment 3, we repeated the results of Experiment 1 and 2 with a within-subject experimental design. The results rejected the possibility that the negative emotional effect shown in Experiment 2 was due to a longer time of emotion induction. In addition, Experiment 3 showed that there was no significant negative emotional effect under the short presentation time condition, while under long presentation time 
condition, the negative emotional state had a significant effect on the number and precision of VWM. These results supported our hypothesis that the influence of the negative emotional state on VWM representation was related to resource allocation in the late VWM consolidation phase, instead of the early process.

\section{General discussion}

The present study used recall tasks to investigate the impact of the negative emotional state on the number and precision of VWM. The results showed that the negative emotional state improved VWM precision and reduced VWM number, but this effect only occurred when the stimuli were presented for long presentation time. The negative emotional state could not affect the number and precision of VWM when the presentation time of stimuli was short. This suggested that the mechanism underlying the impact of the negative emotional state on VWM was related to the resource allocation in the late VWM consolidation phase instead of the early process.

In fact, in the field of emotion and VWM, besides the influence of the emotional state on VWM, there were also some studies which directly explored the effect of the emotional content on VWM performance (Linden, Jackson, Subramanian, Healy, \& Linden, 2011; Sessa et al., 2011; Ye et al., 2018). These studies showed that participants could remember more negative emotional stimuli than neutral stimuli. This bias toward emotional stimuli may enhance the ability of emotion processing. Thus, participants could memorize more information of the negative content (e.g., angry faces) than that of the neutral content (Jackson, Linden, \& Raymond, 2014; Jackson, Wu, Linden, \& Raymond, 2009). However, because there are different sensory attributes between negative emotional stimuli and neutral stimuli, the studies of the effects of the emotional content on VWM would inevitably mix with the attention bias (Maljkovic \& Martini, 2005). For example, negative images were more likely to attract attention than neutral images (Phelps, Ling, \& Carrasco, 2006). Therefore, in order to avoid this confusion, the present study only manipulated the emotional state while keeping the memory content as the simple orientations to ensure that the influence of the emotional state on VWM was mainly related to the late phase of resource allocation in VWM consolidation, but not to the early process.

Although our study avoided the effect of memory content on VWM, our results were not entirely consistent with all previous studies on the influence of the negative emotional state on VWM. The results of our Experiment 2 and 3 suggested that when the presentation time was extended to $500 \mathrm{~ms}$, the negative emotional state could lead to an increase of VWM precision and a decrease of VWM number. By calculating the experimental effects of the negative emotional state, we found that there was no significant difference in the increase of VWM precision $(16.77 \%)$ and decrease of VWM number $(18.27 \%)$ between negative and neutral emotional state conditions. That is, the negative emotional state affects both VWM precision and VWM number in the long presentation time condition. Therefore, our results supported that the 
negative emotion affected the trade-off between number and precision of VWM, rather than affecting VWM precision alone or VWM number alone. Our results were in line with the results of VWM task in Spachtholz et al. (2014)'s study, and rejected that the negative emotional state could enhance minimum memory precision of VWM representations in the early consolidation phase. However, in Xie and Zhang (2016)'s study, although there was enough presentation time of stimuli for the participants to memorize the items (400 ms for five colors in Exp 1; $1000 \mathrm{~ms}$ for four shapes in Exp 2 ), no evidence supported that the negative emotional state led to the change in trade-off ratio between the VWM number and precision. Their results only suggested that the negative emotional state could improve the VWM precision. One possible explanation for the different conclusions between their results and our results was that, in Xie and Zhang (2016)'s study (Exp 1), the number of items (five items) participants needed to remember was higher than the general VWM capacity (three to four items). A recent study found that when the number of stimuli exceeded the upper limit of individual VWM capacity, the memory performance of the individuals was worse than when they need to memorize items of a near-capacity set size (Fukuda, Mance, \& Vogel, 2015). In Xie and Zhang (2016)'s study, although there was enough time to memorize the stimuli $(400 \mathrm{~ms})$, participants could not consolidate all stimuli into VWM because of the limitation of VWM capacity. This may lead to extra interference, which may reduce VWM number. Since the negative emotion was found to narrow attention focus (Fenske \& Eastwood, 2003; Finucane, 2011), it may reduce the interference when participants consolidated the stimuli of supra-capacity set sizes. However, participants automatically traded VWM number for VWM precision in the negative emotional state. In this case, the trade-off between VWM number and precision did not necessarily lead to a change in $\mathrm{K}$ value. Because although the $\mathrm{K}$ value decreased in the trade-off, it also increased as interference decrease. Therefore, although there were significant differences in VWM precision between the different emotional state conditions, there was no difference in $\mathrm{K}$ value between the conditions. This possibility could also explain the result difference between Xie and Zhang (2016)'s study and Spachtholz et al. (2014)'s study. We needed to point out that our findings did not necessarily challenge the results of Xie and Zhang (2016)"s study. On the contrary, combining with the previous evidence, we proposed that the negative emotional state might have different effects on VWM performance under different memory loads. Future research could further test this hypothesis.

It is worth noting that the aim of our study was to test whether the impact of the negative emotional state on VWM was related to the early phase or late phase of VWM consolidation process. In the study of Spachtholz et al. (2014), they also examined the effect of the negative emotional state on VSM. They found that the negative emotion can also improve precision and reduced number of VSM, which suggested that negative emotion affected the sensory encoding process. At first glance, our results are in contrast to Spachtholz et al. (2014)'s study. These discrepant findings can be explained by the difference in the experimental design (memory set size) between these two studies. In their VSM task, participants needed to remember 
six items. Participants in a negative emotional state may narrow their attention focus to reduce the amount number of VSM. Therefore, in their VSM tasks, we can observe that emotion modulated memory representations even in the early sensory memory state. However, in our study, participants only needed to remember four items. The results of Experiment 1 and Experiment 3 in our study showed that negative emotional state does not modulate memory representations in the early consolidation phase or other early processes (e.g., sensory encoding process). It suggested that when the participants only need to memorize four items, even though the negative emotional state may narrow attention focus, they still have enough attention span to cover all items. Nevertheless, our study suggested that the effect of emotional state on VWM representations can be independent of the early process, but our study could not be used to reject the effect of emotional state on sensory encoding process. Especially when the encoding set size is large, the negative emotional state could have a significant impact on sensory encoding processes.

In our study, we applied bilateral recall tasks to enable participants to allocate VWM resources effectively (Y. Zhang et al., 2018). Although previous studies have found the negative emotion could lead to a narrow attention state (Finucane, 2011), their results could not reject the results of our study. The tasks in most of the studies on visual attention did not involve the processing of VWM. In the present study, we distinguished different processes based on the predictions of the two-phase model (Ye et al., 2017; Ye et al., 2019). The results showed that the negative emotional state affected the late phase of resource allocation in the consolidation process. These findings were in line with Li, Chan, and Luo (2010)'s study. They used a delayed matching-to-sample task to investigate the impact of the negative emotional state on the verbal working memory and visuospatial working memory. They found that the negative emotional state led to worse memory performance compared with the neutral emotional state. More importantly, their ERP results suggested that the difference between the negative emotional state and neutral emotional state was mainly in the VWM maintenance stage, which implied that the negative emotional state had distinct stages of influence on VWM, and there was a systematic link between specific emotions and certain cognitive processes. Future studies can investigate the effects of different emotional states on resource allocation during memory maintenance, based on VWM studies with the retro-cue (Griffin \& Nobre, 2003; Landman, Spekreijse, \& Lamme, 2003).

It is worth noting that the results of Experiment 3 show that especially in the neutral emotional state the longer presentation time did not lead to a substantial influence on VWM precision, even though it increased $\mathrm{K}$ value. This result might be due to two aspects. The first aspect was that in the neutral emotional state the VWM capacity limitation of most participants would be reached when four orientation are memorized. Thus, participants would memorize the items as many as possible with the lowest precision. The second aspect was that the consolidation of orientation is a serial consolidation process (Becker et al., 2013; Hao et al., 2018; Liu \& Becker, 2013; 
Miller et al., 2014). The consolidation speed of participants was about $100 \mathrm{~ms} / \mathrm{item}$ (Vogel, Woodman, \& Luck, 2006). Thus, in the short presentation time condition, the number of items consolidated should be less than the upper limit of their VWM capacity. In contrast, in the long presentation time condition, the number of items consolidated would reach the upper limit. However, no matter in short or long presentation time conditions, participants would store items with the same minimum precision. In the neutral emotional state, participants would not sacrifice VWM number to improve VWM precision. Therefore, the VWM number increased with the presentation time but the VWM precision remains the same in the neutral emotional state.

It is natural to ask whether different emotional states really modulate VWM resource allocation or manipulate the generic task strategies of participants under different emotional conditions (e.g., strategically trade quantity for quality only in the negative emotional condition). By mixing different emotional conditions in each block, Experiment 2 in Xie and Zhang (2016)'s study provided evidence that the effect of emotional modulation was not due to the difference in generic task strategy. Moreover, in Experiment 3, we mixed different presentation time conditions in each emotional block. We found that there were differences in trade-off patterns under different presentation time conditions in the same emotional block. More importantly, participants who used different generic task strategies under different emotional conditions had no obvious benefit. The difference in generic task strategy under different emotional blocks seems difficult to explain these results. It is possible that the trade-off of precision and capacity was based on automatic resource allocation (i.e., cognitive process bias). When individuals are able to reallocate VWM resource (at the late phase of VWM resource allocation), they may have an involuntary bias to trade quantity for quality under the negative emotional state. This involuntary bias for quality might have evolved because of the need to maintain more detailed information to deal with potential dangers that often arise in negative situations. Previous studies could support this idea. For example, Sessa et al. (2011)'s study has shown that the VWM precision of fearful face is higher than that of the neutral face.

A recent study has shown that in the recall task, the response data may show categorical response biases (Hardman, Vergauwe, \& Ricker, 2017). Thus, it is possible that the difference of trade-off patterns between VWM precision and number in different emotional states was due to the effect of negative emotional state on categorical response biases. That is, the emotional state modulates the tendency to make a response based on categorical information. However, this possibility could not explain why the negative emotional state could not affect the trade-off in the short presentation time (200 ms) condition. In addition, we checked our dataset by visual observation. The only interesting thing was that when the participants made a guess response (reported angle and actual angle were very different), they may choose a 90-degree angle, but they would choose less near 90 degrees (e.g., 85-89 degree or 91-95 degree). This may be because the 90-degree angle (vertical angle) was the 
default value in our experiments. When participants decided to choose a vertical angle to make a guess response, they were not willing to make further adjustments to the angle. However, this phenomenon existed in both neutral and negative emotional state conditions (in both short and long presentation time conditions). Therefore, although our participants may have a certain bias (especially for vertical angles) in response, this might not be the main reason to cause the difference between different emotional state conditions in our study.

In our study, as Ye et al. (2017)'s study, we used $200 \mathrm{~ms}$ and $500 \mathrm{~ms}$ presentation time (without a post-mask) to distinguish different presentation time conditions. Previous studies found that, because of retinal persistence, visual information persistence can exist $100 \mathrm{~ms}$ to $500 \mathrm{~ms}$ after stimulus disappeared (Averbach \& Coriell, 1961; Brockmole, Wang, \& Irwin, 2002; Coltheart, 1980; Di Lollo \& Dixon, 1988). It seems to suggest that there is little difference between different presentation time conditions. However, it may not be the case. After the memory items disappear, individuals could use the retinal persistence to consolidate representations, but when they needed to improve memory precision through consolidation, it mainly relies on the period when the external stimulus still exists, instead of the total time when visual information of retinal persistence exists. This was because only when the external stimulus still exists can individuals extract more detailed information from the external visual stimulus for consolidation to improve the precision of VWM representation. Once the visual stimulus disappears, although the visual information could still exist on the retina for a period of time, the visual information fades rapidly during this period, which makes it difficult for consolidation to use the visual information of retinal persistence to improve VWM precision. Therefore, the visual information of retinal persistence does not weaken the effect of stimulus presentation time on VWM representations. Although some early studies on VWM consolidation mainly used fixed presentation time of memory array and manipulated the memory-and-mask ISI (Vogel et al., 2006; W. Zhang \& Luck, 2008), recent studies on VWM consolidation had begun to directly manipulate the presentation time of memory array with no memory-and-mask ISI (Becker et al., 2013; Hao et al., 2018; Liu \& Becker, 2013; Mance et al., 2012; Miller et al., 2014; Rideaux, Apthorp, \& Edwards, 2015; Rideaux \& Edwards, 2016). Therefore, in our study, the presentation time of memory array not only limits the encoding process, but also limits the time that participants can improve memory precision in VWM consolidation. However, because different researchers may have some subtle differences in the definition of VWM consolidation, this may lead to uncertainty in the relationship between presentation time manipulation and VWM consolidation. In particular, the presentation time may have an impact on some processing (e.g., eye-movement, mind wandering) other than VWM. Future studies need to pay attention to the influence of these other time-related factors on the results when researchers control the presentation time of memory array.

Recently, Xie and Zhang (2017) found that negative emotions improved not only the 
VWM precision but also the precision of visual long-term memory (VLM). The mechanism underlying the impact of the negative emotional state on VLM may be different from that of the negative emotion on VWM. Future studies can compare the effects of negative emotion on VWM and VLM to investigate the effects of emotional states on memory mechanisms. In addition, the research on the effect of emotional state on VWM can not be limited to memory storage. For example, by using a retro-cue that points to an object (Griffin \& Nobre, 2003; Landman et al., 2003) or a dimension (Niklaus, Nobre, \& van Ede, 2017; Ye, Hu, Ristaniemi, Gendron, \& Liu, 2016), previous studies found that attention can be directed to information in VWM and improve the VWM performance. This phenomenon is called the retro-cue effect in VWM. Future studies can examine the effect of different emotional states on the retro-cue effect.

It is also worth noting that there might be some model fitting artifacts in our results due to a limited number of trials per condition. Therefore, although our study proposed a new perspective to reconcile the conflicting findings of previous relevant studies (Spachtholz et al., 2014; Xie \& Zhang, 2016), the conclusion needs to be further confirmed in future studies. Moreover, our results have provided new evidence for the effect of emotional state at the behavioral level. Future studies need to leverage converging evidence from both behavioral and neural data (e.g., EEG, MEG) to better articulate this issue.

In summary, our study found that the effect of negative emotional state on the trade-off between quantity and quality of VWM may only occur under the condition with a long presentation time of the memory stimuli. These results suggested that the emotional modulation of VWM mainly related to the late phase of VWM resource allocation in the consolidation process, instead of in the early consolidation phase. By affecting the late phase of VWM resource allocation, the negative emotional state could improve VWM precision by sacrificing VWM number. The findings from this study make important contributions to the current literature regarding the emotional modulation of VWM.

\section{Acknowledgments}

We would like to thank Dr. Weizhen Xie and Ms. Qianru Xu for the helpful discussions. We also want to thank Dr. Tengfei Liang and Ms. Xiaoyu Chen for their valuable contribution to the data acquisition. Finally, we also want to thank two anonymous reviewers for providing constructive feedback in the review process.

\section{Author Contributions Statement}

F. L., Q. L., and C. Y. conceived and designed the experiments. F. L., performed the data acquisition and analyzed the data. Q. L., C.Y., and F. L. interpreted the data. C. Y., F. L., and Q. L. drafted the manuscript. All authors revised and approved the 
manuscript.

\section{Conflict of interest}

The authors have declared that no competing financial and/or non-financial interests exist.

\section{Data availability statement}

The datasets generated during and/or analysed during the current study are available from the corresponding author (lq780614@163.com, Qiang Liu) on reasonable request. 


\section{Reference}

Averbach, E., \& Coriell, A. S. (1961). Short-term memory in vision. Bell System Technical Journal, 40(1), 309-328.

Balaban, H., Fukuda, K., \& Luria, R. (2019). What can half a million change detection trials tell us about visual working memory? Cognition, 191, 103984. doi: 10.1016/j.cognition.2019.05.021

Barton, B., Ester, E. F., \& Awh, E. (2009). Discrete resource allocation in visual working memory. $J$ Exp Psychol Hum Percept Perform, 35(5), 1359-1367. doi: 10.1037/a0015792

Bays, P. M., Catalao, R. F., \& Husain, M. (2009). The precision of visual working memory is set by allocation of a shared resource. $J$ Vis, 9(10), 7 1-11. doi: 10.1167/9.10.7

Bays, P. M., \& Husain, M. (2008). Dynamic shifts of limited working memory resources in human vision. Science, 321(5890), 851-854. doi: 10.1126/science.1158023

Bays, P. M., Wu, E. Y., \& Husain, M. (2011). Storage and binding of object features in visual working memory. Neuropsychologia, 49(6), 1622-1631. doi: 10.1016/j.neuropsychologia.2010.12.023

Becker, M. W., Miller, J. R., \& Liu, T. (2013). A severe capacity limit in the consolidation of orientation information into visual short-term memory. Atten Percept Psychophys, 75(3), 415-425. doi: 10.3758/s13414-012-0410-0

Brockmole, J. R., Wang, R. F., \& Irwin, D. E. (2002). Temporal integration between visual images and visual percepts. J Exp Psychol Hum Percept Perform, 28(2), 315-334.

Coltheart, M. (1980). Iconic memory and visible persistence. Percept Psychophys, 27(3), 183-228.

Di Lollo, V., \& Dixon, P. (1988). Two forms of persistence in visual information processing. J Exp Psychol Hum Percept Perform, 14(4), 671-681.

Faul, F., Erdfelder, E., Lang, A. G., \& Buchner, A. (2007). G*Power 3: a flexible statistical power analysis program for the social, behavioral, and biomedical sciences. Behav Res Methods, 39(2), 175-191.

Fenske, M. J., \& Eastwood, J. D. (2003). Modulation of focused attention by faces expressing emotion: evidence from flanker tasks. Emotion, 3(4), 327-343. doi: 10.1037/1528-3542.3.4.327

Figueira, J. S. B., Oliveira, L., Pereira, M. G., Pacheco, L. B., Lobo, I., Motta-Ribeiro, G. C., \& David, I. A. (2017). An unpleasant emotional state reduces working memory capacity: electrophysiological evidence. Soc Cogn Affect Neurosci, 12(6), 984-992. doi: 10.1093/scan/nsx030

Figueira, J. S. B., Pacheco, L. B., Lobo, I., Volchan, E., Pereira, M. G., de Oliveira, L., \& David, I. A. (2018). "Keep That in Mind!" The Role of Positive Affect in Working Memory for Maintaining Goal-Relevant Information. Front Psychol, 9, 1228. doi: 10.3389/fpsyg.2018.01228

Finucane, A. M. (2011). The effect of fear and anger on selective attention. Emotion, 11(4), 970-974. doi: $10.1037 / \mathrm{a} 0022574$

Fukuda, K., Mance, I., \& Vogel, E. K. (2015). a power modulation and event-related slow wave provide dissociable correlates of visual working memory. J Neurosci, 35(41), 14009-14016. doi: 10.1523/Jneurosci.5003-14.2015

Fukuda, K., Vogel, E., Mayr, U., \& Awh, E. (2010). Quantity, not quality: the relationship between fluid intelligence and working memory capacity. Psychon Bull Rev, 17(5), 673-679. doi: 10.3758/17.5.673

Gao, Z., Yin, J., Xu, H., Shui, R., \& Shen, M. (2011). Tracking object number or information load in 
visual working memory: revisiting the cognitive implication of contralateral delay activity. Biol Psychol, 87(2), 296-302. doi: 10.1016/j.biopsycho.2011.03.013

Griffin, I. C., \& Nobre, A. C. (2003). Orienting attention to locations in internal representations. J Cogn Neurosci, 15(8), 1176-1194. doi: 10.1162/089892903322598139

Hao, R., Becker, M. W., Ye, C., Liu, Q., \& Liu, T. (2018). The bandwidth of VWM consolidation varies with the stimulus feature: Evidence from event-related potentials. J Exp Psychol Hum Percept Perform, 44(5), 767-777. doi: 10.1037/xhp0000488

Hardman, K. O., Vergauwe, E., \& Ricker, T. J. (2017). Categorical working memory representations are used in delayed estimation of continuous colors. J Exp Psychol Hum Percept Perform, 43(1), 30-54. doi: 10.1037/xhp0000290

He, X., Zhang, W., Li, C., \& Guo, C. (2015). Precision requirements do not affect the allocation of visual working memory capacity. Brain Res, 1602, 136-143. doi: 10.1016/j.brainres.2015.01.028

Ikkai, A., McCollough, A. W., \& Vogel, E. K. (2010). Contralateral delay activity provides a neural measure of the number of representations in visual working memory. J Neurophysiol, 103(4), 1963-1968. doi: 10.1152/jn.00978.2009

Jackson, M. C., Linden, D. E. J., \& Raymond, J. E. (2014). Angry expressions strengthen the encoding and maintenance of face identity representations in visual working memory. Cogn Emot, 28(2), 278-297. doi: 10.1080/02699931.2013.816655

Jackson, M. C., Wu, C. Y., Linden, D. E., \& Raymond, J. E. (2009). Enhanced visual short-term memory for angry faces. J Exp Psychol Hum Percept Perform, 35(2), 363-374. doi: $10.1037 / \mathrm{a} 0013895$

Landman, R., Spekreijse, H., \& Lamme, V. A. (2003). Large capacity storage of integrated objects before change blindness. Vision Res, 43(2), 149-164.

Li, X., Chan, R. C., \& Luo, Y. J. (2010). Stage effects of negative emotion on spatial and verbal working memory. BMC Neurosci, 11, 60. doi: 10.1186/1471-2202-11-60

Linden, S. C., Jackson, M. C., Subramanian, L., Healy, D., \& Linden, D. E. (2011). Sad benefit in face working memory: an emotional bias of melancholic depression. J Affect Disord, 135(1-3), 251-257. doi: 10.1016/j.jad.2011.08.002

Liu, T., \& Becker, M. W. (2013). Serial consolidation of orientation information into visual short-term memory. Psychol Sci, 24(6), 1044-1050. doi: 10.1177/0956797612464381

Luck, S. J., \& Vogel, E. K. (1997). The capacity of visual working memory for features and conjunctions. Nature, 390(6657), 279-281. doi: 10.1038/36846

Luck, S. J., \& Vogel, E. K. (2013). Visual working memory capacity: from psychophysics and neurobiology to individual differences. Trends Cogn Sci, 17(8), 391-400. doi: 10.1016/j.tics.2013.06.006

Luria, R., Balaban, H., Awh, E., \& Vogel, E. K. (2016). The contralateral delay activity as a neural measure of visual working memory. Neurosci Biobehav Rev, 62, 100-108. doi: 10.1016/j.neubiorev.2016.01.003

Ma, W. J., Husain, M., \& Bays, P. M. (2014). Changing concepts of working memory. Nat Neurosci, 17(3), 347-356. doi: 10.1038/nn.3655

Machizawa, M. G., Goh, C. C., \& Driver, J. (2012). Human visual short-term memory precision can be varied at will when the number of retained items is low. Psychol Sci, 23(6), 554-559. doi: $10.1177 / 0956797611431988$ 
Maljkovic, V., \& Martini, P. (2005). Short-term memory for scenes with affective content. J Vis, 5(3), 215-229. doi: $10.1167 / 5.3 .6$

Mance, I., Becker, M. W., \& Liu, T. (2012). Parallel consolidation of simple features into visual short-term memory. J Exp Psychol Hum Percept Perform, 38(2), 429-438. doi: $10.1037 / \mathrm{a} 0023925$

Miller, J. R., Becker, M. W., \& Liu, T. (2014). The bandwidth of consolidation into visual short-term memory depends on the visual feature. Vis cogn, 22(7), 920-947. doi: $10.1080 / 13506285.2014 .936923$

Niklaus, M., Nobre, A. C., \& van Ede, F. (2017). Feature-based attentional weighting and spreading in visual working memory. Sci Rep, 7, 42384. doi: 10.1038/srep42384

Phelps, E. A., Ling, S., \& Carrasco, M. (2006). Emotion facilitates perception and potentiates the perceptual benefits of attention. Psychol Sci, 17(4), 292-299. doi: 10.1111/j.1467-9280.2006.01701.x

Rideaux, R., Apthorp, D., \& Edwards, M. (2015). Evidence for parallel consolidation of motion direction and orientation into visual short-term memory. $J$ Vis, 15(2), 17.

Rideaux, R., \& Edwards, M. (2016). The cost of parallel consolidation into visual working memory. $J$ Vis, 16(6). doi: 10.1167/16.6.1

Sessa, P., Luria, R., Gotler, A., Jolicoeur, P., \& Dell'Acqua, R. (2011). Interhemispheric ERP asymmetries over inferior parietal cortex reveal differential visual working memory maintenance for fearful versus neutral facial identities. Psychophysiology, 48(2), 187-197. doi: 10.1111/j.1469-8986.2010.01046.x

Spachtholz, P., Kuhbandner, C., \& Pekrun, R. (2014). Negative affect improves the quality of memories: trading capacity for precision in sensory and working memory. J Exp Psychol Gen, 143(4), 1450-1456. doi: 10.1037/xge0000012

Spies, K., Hesse, F., \& Hummitzsch, C. (1996). Mood and capacity in Baddeley's model of human memory. Zeitschrift für Psychologie mit Zeitschrift für angewandte Psychologie.

Suchow, J. W., Brady, T. F., Fougnie, D., \& Alvarez, G. A. (2013). Modeling visual working memory with the MemToolbox. $J$ Vis, 13(10). doi: 10.1167/13.10.9

Suchow, J. W., Fougnie, D., Brady, T. F., \& Alvarez, G. A. (2014). Terms of the debate on the format and structure of visual memory. Atten Percept Psychophys, 76(7), 2071-2079. doi: $10.3758 / \mathrm{s} 13414-014-0690-7$

Thalheimer, W., \& Cook, S. (2002). How to calculate effect sizes from published research: A simplified methodology. Work-Learning Research, 1, 1-9.

Vogel, E. K., \& Machizawa, M. G. (2004). Neural activity predicts individual differences in visual working memory capacity. Nature, 428(6984), 748-751. doi: 10.1038/nature02447

Vogel, E. K., Woodman, G. F., \& Luck, S. J. (2001). Storage of features, conjunctions and objects in visual working memory. J Exp Psychol Hum Percept Perform, 27(1), 92-114.

Vogel, E. K., Woodman, G. F., \& Luck, S. J. (2006). The time course of consolidation in visual working memory. $J$ Exp Psychol Hum Percept Perform, 32(6), 1436-1451. doi: 10.1037/0096-1523.32.6.1436

Wilken, P., \& Ma, W. J. (2004). A detection theory account of change detection. J Vis, 4(12), 1120-1135. doi: 10:1167/4.12.11

Xie, W., \& Zhang, W. (2016). Negative Emotion Boosts Quality of Visual Working Memory Representation. Emotion, 16(5), 760-774. doi: 10.1037/emo0000159 
Xie, W., \& Zhang, W. (2017). Negative emotion enhances mnemonic precision and subjective feelings of remembering in visual long-term memory. Cognition, 166, 73-83. doi: 10.1016/j.cognition.2017.05.025

Ye, C., Hu, Z., Li, H., Ristaniemi, T., Liu, Q., \& Liu, T. (2017). A two-phase model of resource allocation in visual working memory. J Exp Psychol Learn Mem Cogn, 43(10), 1557-1566. doi: $10.1037 / x \operatorname{lm} 0000376$

Ye, C., Hu, Z., Ristaniemi, T., Gendron, M., \& Liu, Q. (2016). Retro-dimension-cue benefit in visual working memory. Sci Rep, 6, 35573. doi: 10.1038/srep35573

Ye, C., Sun, H. J., Xu, Q., Liang, T., Zhang, Y., \& Liu, Q. (2019). Working memory capacity affects trade-off between quality and quantity only when stimulus exposure duration is sufficient: Evidence for the two-phase model. Sci Rep, 9(1), 8727. doi: 10.1038/s41598-019-44998-3

Ye, C., Xu, Q., Liu, Q., Cong, F., Saariluoma, P., Ristaniemi, T., \& Astikainen, P. (2018). The impact of visual working memory capacity on the filtering efficiency of emotional face distractors. Biol Psychol, 138, 63-72. doi: 10.1016/j.biopsycho.2018.08.009

Ye, C., Zhang, L., Liu, T., Li, H., \& Liu, Q. (2014). Visual working memory capacity for color is independent of representation resolution. PLoS One, 9(3), e91681. doi: 10.1371/journal.pone.0091681

Zhang, W., \& Luck, S. J. (2008). Discrete fixed-resolution representations in visual working memory. Nature, 453(7192), 233-235. doi: 10.1038/nature06860

Zhang, Y., Ye, C., Roberson, D., Zhao, G., Xue, C., \& Liu, Q. (2018). The bilateral field advantage effect in memory precision. $Q J$ Exp Psychol (Hove), 71(3), 749-758. doi: 10.1080/17470218.2016.1276943

Zhang, Y., Zhang, G., \& Liu, B. (2017). Investigation of the influence of emotions on working memory capacity using ERP and ERSP. Neuroscience, 357, 338-348. doi: 10.1016/j.neuroscience.2017.06.016 